www.nature.com/ejhg

\title{
Cryptic subtelomeric $6 p$ deletion in a girl with congenital malformations and severe language impairment
}

\author{
Britt-Marie Anderlid ${ }^{*}{ }^{1}$, Jacqueline Schoumans ${ }^{1}$, Åsa Hallqvist ${ }^{1}$, Ylva Ståhl ${ }^{2}$, \\ Agneta Wallin $^{3}$, Elisabeth Blennow ${ }^{1}$ and Magnus Nordenskjöld ${ }^{1}$ \\ ${ }^{1}$ Department of Molecular Medicine, Clinical Genetics Unit, Karolinska Institutet at Karolinska Hospital, Stockholm, \\ Sweden; ${ }^{2}$ Department of Neuropediatrics, Astrid Lindgrens Children's Hospital, Karolinska Hospital, Stockholm, \\ Sweden; ${ }^{3}$ Department of Pediatric Ophtalmology and Strabismus, Danderyds Hospital, St Eriks Eye Hospital, \\ Stockholm, Sweden
}

Several cases with microscopically visible, terminal $6 \mathrm{p}$ deletions have been described, and a distinct clinical phenotype has emerged, including developmental delay, congenital heart malformations, ocular abnormalities, hearing loss and a characteristic facial appearance. We report a patient with a submicroscopic $6 \mathrm{p}$ deletion, detected by subtelomeric screening using fluorescence in situ hybridisation. This girl presented with typical facial dysmorphic features, hearing impairment, malformation of the anterior eye segment, an ASD and severe language impairment. However, her cognitive functions were within the normal range. Detailed FISH analysis with 20 BAC probes covering the distal $6 \mathrm{p} 25$ region estimated the size of the terminal deletion to $2.1 \mathrm{Mb}$, and thus this case narrows down the critical region for the $6 \mathrm{p}$ phenotype. The forkhead transcription factor gene $\mathrm{FOXC1}$, involved in a spectrum of anterior eye chamber disorders, is deleted in this patient, together with several characterised and putative genes with yet unknown function.

European Journal of Human Genetics (2003) 11, 89-92. doi:10.1038/sj.ejhg.5200907

Keywords: 6p; deletion; subtelomeric; cryptic; language impairment; FISH

\section{Introduction}

Several cases with microscopically visible deletions of the short arm of chromosome 6 have been reported ${ }^{1-3}$ and distinct clinical phenotypes have emerged. The deletions can be grouped into two separate categories: terminal deletions with breakpoints within the 6p24-pter region, and interstitial deletions with breakpoints within the 6p22-p24 region. ${ }^{3}$ The terminal deletions do not seem to overlap with the region of the interstitial deletions, and the clinical phenotypes differ significantly. Short neck, hand abnormalities, structural eye abnormalities, brain, kidney and heart defects are commonly associated with the interstitial deletions, and defects of the anterior eye-chamber

\footnotetext{
*Correspondence: B-M Anderlid, Dept of Molecular Medicine, CMM L8:02, Karolinska Hospital SE-171 76 Stockholm, Sweden.

Tel: +46 851772521 ; Fax: +46 851773620 ;

E-mail: britt.marie.anderlid@cmm.ki.se

Received 24 May 2002; revised 2 September 2002; accepted 23 September 2002
}

development, hearing loss, heart malformations, hypertelorism, mid-face hypoplasia and low set ears are found among the terminal deletions. Developmental delay has been described in all cases.

Cryptic rearrangements of the subtelomeric regions are detected in a significant proportion of cases with idiopathic mental retardation, ${ }^{4}$ and with the possibility to screen all subtelomeric regions with fluorescence in situ hybridisation (FISH), a number of cases with cryptic rearrangements involving the subtelomeric regions has been described.

We here report a patient with a $2.1 \mathrm{Mb}$ subtelomeric deletion of $6 \mathrm{p}$. This is the smallest $6 \mathrm{p}$ deletion hitherto reported, and this case narrows down the critical region and identifies potential candidate genes for the terminal $6 \mathrm{p}$ deletion phenotype. Furthermore, the language impairment present in this case implies the presence of a gene important for speech development in the deleted region. 


\section{Materials and methods}

\section{Case report}

This girl was the second of three children born to healthy, unrelated parents. She had an older healthy sister, and a younger sister was recently born. The father had a paternal cousin with Prader-Willi syndrome, but there were no other cases with developmental delay or malformations in the family history. The pregnancy and delivery was uneventful (GA 40 weeks, BW $4300 \mathrm{~g}$, BL $53 \mathrm{~cm}, \mathrm{HC} 36.6 \mathrm{~cm}$ ). An ASD was diagnosed in the neonatal period, and resulted in feeding difficulties and growth retardation. Cardiac surgery was performed at 18 months and after the operation, feeding normalised and she had a good catch-up in growth $(-1$ SD for length and weight). The motor development had been delayed, and the girl started to walk at age 2 years. However, at 4 years and 9 months of age her motor functions were within the normal range. During the first years of life she had repeated upper airway and ear infections, and an obvious delay in speech development was noticed at age 2 years. Repeated audiometry showed a conductive hearing defect on the right side $(70 \mathrm{~dB})$ and values within the normal range on the left side. Language evaluation disclosed a moderate to severe semantic, pragmatic language impairment, in particular in understanding phrases and concepts and difficulties in explaining and relating. She had no articulation problems but difficulties in the social interaction of speech and a lack in eye contact. Her psychological functions were tested with the Leiter-R scale at age 4 years and 9 months, and her results were average with the best results in visual tasks. She had a convergent squint, hyperopia (+6 dioptres), a deep anterior chamber and goniodysgenesis in combination with a normal intra-ocular pressure. Several dysmorphic features were present (Figure 1): hypertelorism, long, downward slanting palpebral fissures, bilateral epicantus, flat and broad base of the nose, short nose with upturned tip, long smooth philtrum, thin upper-lip without a cupid bow, down turned corners of the mouth, low set ears, prominent and cone-shaped canine teeth (both upper and lower jaw) and short fingers and toes.

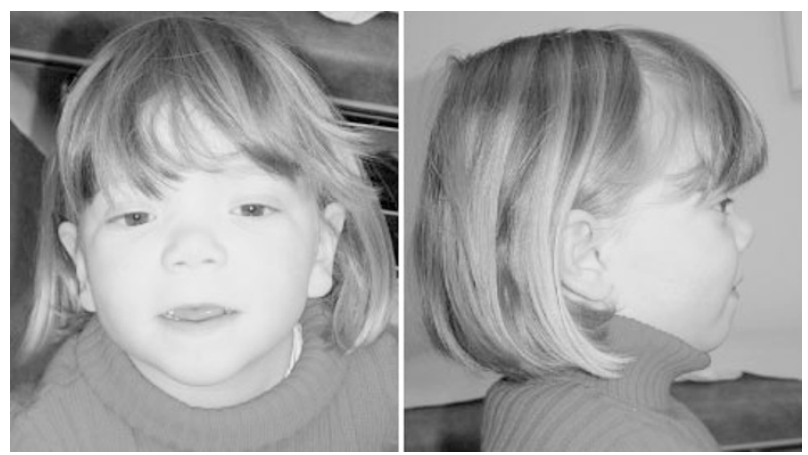

Figure 1 Facial appearance of the patient at age 4 years and 9 months.
Conventional chromosome analysis (450 band resolution) was performed, but revealed no abnormalities.

\section{Cytogenetic studies}

Metaphase slides were prepared from lymphocyte cultures of peripheral blood. Chromosome analysis was performed according to routine procedures using GTG-banding.

\section{Fluorescence in situ hybridisation (FISH)}

The ToTelVysion subtelomeric screening kit (Vysis, Downers Grove, IL, USA) was used to screen the subtelomeric regions. The test included 41 subtelomeric probes estimated to be within $300 \mathrm{~kb}$ from the telomere. The manufacturer's protocol was used with some modifications.

BAC-probes covering 6p25 were selected from Resources for Molecular Cytogenetics, BARI (www.biologia.uniba.it/ $\mathrm{rmc} /$ ) and The Sanger Centre (www.sanger.ac.uk). Bacterial cultures and DNA isolation were performed according to a miniprep protocol. Probes were labelled with FITC by nick translation, and FISH-analyses were performed according to a standard protocol.

The slides were analysed in a Zeiss Axioscope II fluorescence microscope (Zeiss, Göttingen, Germany) and images captured by a cooled CCD camera (Sensys Photometrix, München, Germany) and the software SmartCapture (Digital Scientific Ltd, Cambridge, UK). Inverted DAPI staining was used for chromosome identification during FISH analysis.

\section{Results}

The subtelomeric screening disclosed an isolated deletion of chromosome $6 \mathrm{p}$. Repeated chromosome analysis with high resolution (600 band) could not reveal the deletion. FISH with a subtelomeric $6 \mathrm{p}$ probe (TelVysion 6p, Vysis) was normal in both parents, thus the deletion was de novo. The mother was 13 weeks pregnant at the time of investigation and prenatal chromosome and FISH-analyses were performed, both with normal results.

In order to determine the size and confirm the terminal location of the deletion, the hybridisation patterns of 20 BAC clones from distal 6 p25 were evaluated. The results from nine clones located within $4 \mathrm{Mb}$ of the telomere are summarised in Figure 2. The breakpoint was located between the contiguous clones 27919 (deleted) and 82M9 (not deleted), and the size of the deletion was estimated to $2.1 \mathrm{Mb}$ (Ensembl Human Contig View, www.ensembl. org/).

\section{Discussion}

The clinical phenotype in terminal $6 \mathrm{p}$ deletions is well described, and most of the symptoms are present in this case, although this deletion is smaller than those previously reported. Knight et al. reported a case with a $6 \mathrm{p}$ deletion estimated to $6.4-8.6 \mathrm{~cm}$, but did not include clinical data beside mental retardation. ${ }^{4}$ Davies et al. ${ }^{3}$ compared the clin- 


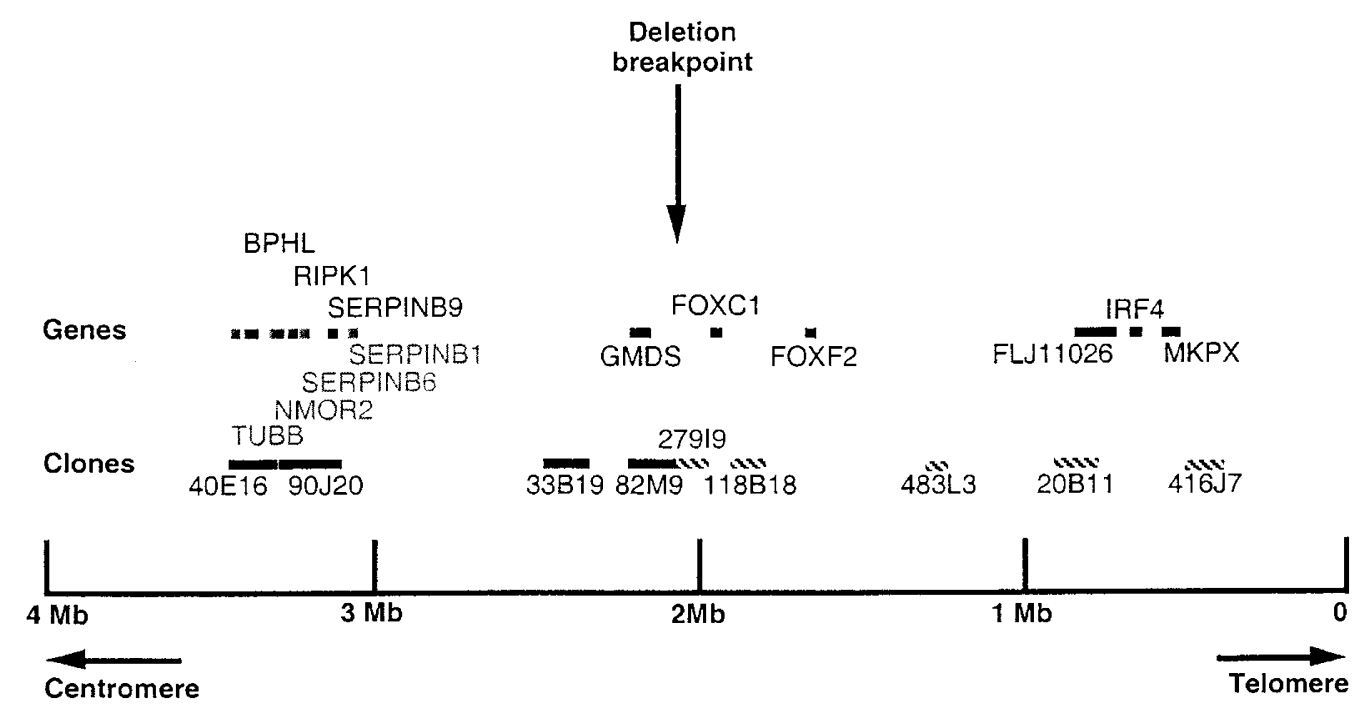

Figure 2 Mapping of the $6 \mathrm{p}$ deletion. The picture summarises the FISH analysis with BAC probes and shows the localisation of the characterised genes in the region. Genes are shown as horizontal bars (top). Deleted BAC clones are pictured striped and present BAC clones in black. The vertical arrow indicates the deletion breakpoint. Location of BAC's and genes are according to Ensembl Human Contig View.

ical phenotype and mapped three terminal $6 \mathrm{p}$ deletions. The smallest deletion, estimated to $4.8-6.7 \mathrm{Mb}$, could be detected with high-resolution chromosome analysis. That case, earlier reported by Law et al., ${ }^{5}$ suggested a more specific distal $6 \mathrm{p}$ deletion phenotype, including characteristic facies with hypertelorism, a down-turned tented mouth, anterior chamber malformation of the eye, progressive sensineuronal deafness, cardiac defects and speech and motor delay. Our case with a $2.1 \mathrm{Mb}$ distal deletion confirms this phenotype, and further narrows down the critical region for the genes involved.

The deletion encompasses two forkhead/winged helix (FOX) genes, FOXC1 and FOXF2. FOX-genes constitute a family of transcription factors, named by their characteristic DNA-binding domain. This group of genes have turned out to play an important role in embryonic development of different tissues and the establishment of the body axis and midline structures. ${ }^{6,7}$ A correct gene dosage of FOXgenes seems important for embryologic development ${ }^{8,9}$ and mutations in FOX-genes have been found in human developmental disorders such as FOXE1 (thyroid agenesis, cleft palate and choanal atresia ${ }^{10}$ ), FOXC2 (hereditary lymphoedema-distichiasis syndrome ${ }^{11}$ ) and FOXL2 $\left(\mathrm{BPES}^{12}\right.$ ).

Consistent findings in terminal $6 \mathrm{p}$ deletions are abnormalities of the anterior chamber of the eye. Furthermore, several clinically related disorders of anterior-segment formation have been mapped to the IRID1 (OMIM 601631 ) locus in $6 \mathrm{p} 25 .{ }^{13}$ In addition, chromosome aberrations including $6 \mathrm{p} 25$ have been reported together with anterior segment malformations and additional clinical findings. ${ }^{1,3,14}$ The FOX-gene FOXC1 is located in the critical IRID1 region and deleted in our patient, and mutations in this gene have been found in familial and isolated cases with anterior chamber anomalies. However, not all families with anterior chamber defects mapped to $6 \mathrm{p} 25$ have mutations in the FOXC1 gene, which implies the presence of other genes involved in eye development in the region.

In contrast to most previously reported terminal $6 \mathrm{p}$ deletion cases, our case does not have mental retardation, but she has a severe language impairment and difficulties in social interaction. This suggests that a gene involved in the development of speech and reciprocity of interaction could be located in the deleted region. The first gene involved in speech development was recently identified as a FOX-gene, FOXP2. ${ }^{15}$ Mutations in this gene were found in the family $\mathrm{KE},{ }^{16}$ a family in which abnormalities in articulation and language processing segregates as a dominant trait, and in addition the gene was found to be disrupted in a translocation patient with a similar speech defect.

FOXF2 is located distal to FOXC1 and constitutes a candidate gene for the second IRID1 locus. In two different families with duplications of 6 p25 containing both genes, iris hypoplasia has been found, and a proper gene dosage of both FOXC1 and FOXF2 seems important for normal eye development. ${ }^{8}$ The mouse homologue of FOXF2 is expressed in mesenchymal tissues, central nervous system, eye, ear and limb buds during embryogenesis, implying a possible role in the normal development of the eye, ear and nervous system. ${ }^{17}$ FOXF2 is deleted in our patient and constitutes a candidate gene, not only for normal eye development, but also for normal speech development. 
In addition, three other cloned genes and several putative genes are deleted in the present case. IRF4 codes for a transcription factor involved in the transcriptional regulation of interferon and interferon stimulated genes and is highly expressed in spleen and peripheral blood lymphocytes. $^{18}$ The gene FLJ11026, similar to rat rsec, is suggested to have a role in secretory vesicle function. $M K P X$, mitogen activated protein kinas phosphatase $\mathrm{X}$, is a member of a family of kinase phosphatase genes, thought to play a role in the regulation of diverse cellular processes such as proliferation, differentiation and apoptosis through MAP kinases. ${ }^{19}$ The function of the characterised and the putative genes in the deleted region in developmental processes and disease genesis need further elucidation in order to understand the evolution of the $6 p$ deletion phenotype.

To the best of our knowledge, this is the hitherto smallest terminal $6 \mathrm{p}$ deletion reported. Still, in most aspects, the phenotype is concordant to the one found in cases with larger deletions, thus narrowing down the critical region for the localisation of the responsible genes. Several cloned and putative genes are deleted in our patient, and therefore constitute candidate genes for the diverse findings found in the patients with terminal $6 \mathrm{p}$ deletions. Hopefully, future analysis of these genes will give us further knowledge of the genetic influence on speech development and social interaction, hearing impairment and the different malformations seen in the terminal $6 \mathrm{p}$ deletion syndrome.

\section{Acknowledgements}

We thank Prof M Rocchi, Resources for Molecular Cytogenetics, BARI and the Sanger Centre for supplying BAC-probes. This work was supported by funds from the Swedish Medical Research Council, Frimurarna Barnahuset Foundation, Ronald McDonald Child Foundation and Sunnerdahl Foundation.

\section{References}

1 Palmer CG, Bader P, Slovak ML et al: Partial deletion of chromosome 6p: delineation of the syndrome. Am J Med Genet 1991; 39: $155-160$.

2 Davies AF, Olavesen MG, Stephens RJ, Comings DE, Pettenati MJ: A detailed investigation of two cases exhibiting characteristics of the $6 \mathrm{p}$ deletion syndrome. Hum Genet 1996; 98: 454-459.
3 Davies AF, Mirza G, Sekhon G et al: Delineation of two distinct $6 \mathrm{p}$ deletion syndromes. Hum Genet 1999; 104: 64-72.

4 Knight SJ, Regan R, Nicod A et al: Subtle chromosomal rearrangements in children with unexplained mental retardation. Lancet 1999; 354: 1676-1681.

5 Law CJ, Fisher AM, Temple IK: Distal 6p deletion syndrome: a report of a case with anterior chamber eye anomaly and review of published reports. J Med Genet 1998; 35: 685-689.

6 Kaufmann E, Knochel W: Five years on the wings of fork head. Mech Dev 1996; 57: 3-20.

7 Prueitt RL, Zinn AR: A fork in the road to fertility. Nat Genet 2001; 27: $132-134$.

8 Nishimura DY, Searby CC, Alward WL et al: A spectrum of FOXC1 mutations suggests gene dosage as a mechanism for developmental defects of the anterior chamber of the eye. Am J Hum Genet 2001; 68: $364-372$.

9 Smith RS, Zabaleta A, Kume T et al: Haploinsufficiency of the transcription factors FOXC1 and FOXC2 results in aberrant ocular development. Hum Mol Genet 2000; 9: 1021-1032.

10 Clifton-Bligh RJ, Wentworth JM, Heinz P et al: Mutation of the gene encoding human TTF-2 associated with thyroid agenesis, cleft palate and choanal atresia. Nat Genet 1998; 19: 399-401.

11 Fang J, Dagenais SL, Erickson RP et al: Mutations in FOXC2 (MFH1), a forkhead family transcription factor, are responsible for the hereditary lymphedema-distichiasis syndrome. Am J Hum Genet 2000; 67: 1382-1388.

12 Crisponi L, Deiana M, Loi A et al: The putative forkhead transcription factor FOXL2 is mutated in blepharophimosis/ptosis/ epicanthus inversus syndrome. Nat Genet 2001; 27: 159-166.

13 Mears AJ, Jordan T, Mirzayans F et al: Mutations of the forkhead/ winged-helix gene, FKHL7, in patients with Axenfeld-Rieger anomaly. Am J Hum Genet 1998; 63: 1316-1328.

14 Nishimura DY, Swiderski RE, Alward WL et al : The forkhead transcription factor gene FKHL7 is responsible for glaucoma phenotypes which map to 6p25. Nat Genet 1998; 19: 140-147.

15 Lai CS, Fisher SE, Hurst JA, Vargha-Khadem F, Monaco AP: A forkhead-domain gene is mutated in a severe speech and language disorder. Nature 2001; 413: 519-523.

16 Fisher SE, Vargha-Khadem F, Watkins KE, Monaco AP, Pembrey ME: Localisation of a gene implicated in a severe speech and language disorder. Nat Genet 1998; 18: 168-170.

17 Aitola M, Carlsson P, Mahlapuu M, Enerbäck S, Pelto-Huikko M: Forkhead transcription factor FoxF2 is expressed in mesodermal tissues involved in epithelio-mesenchymal interactions. Dev Dyn 2000; 218: 136-149.

18 Grossman A, Mittrucker HW, Nicholl J et al: Cloning of human lymphocyte-specific interferon regulatory factor (hLSIRF/hIRF4) and mapping of the gene to 6p23-p25. Genomics 1996; 37: $229-233$

19 Smith A, Price C, Cullen M et al: Chromosomal localization of three human dual specificity phosphatase genes (DUSP4, DUSP6, and DUSP7). Genomics 1997; 42: 524-527. 